\title{
Genetic and environment factors of mental disorders: Twin studies about Alzheimer's disease, Phobia and Autism Spectrum Disorder
}

\author{
Siyang Wei ${ }^{1, \dagger}$, Zilan $\mathrm{Xin}^{2,{ }^{*}, \dagger}$, and Yuankai Zhang ${ }^{3, \dagger}$ \\ ${ }^{1}$ Columbia Grammar and Preparatory School, NY, USA \\ ${ }^{2}$ Huazhong University of Science and Technology, Wuhan, China \\ ${ }^{3}$ Nanjing Medical University, Jiangsu, China \\ †These authors contributed equally.
}

\begin{abstract}
With the natural and social environment changing, people's mental health is facing severe challenges. Many people are suffering from mental disorders in the present day. A large part of mental disorders can be linked with the nerve system. And nerve system disorders tend to be associated with the development of nerve systems. The gene and the environment can play an important role in the development of the body, and abnormal genes and detrimental environmental factors can contribute a lot to nerve system disorders. Previous research has studied the occurrence and gene-and-environmental impact of many nerve system disorders. Among them, this essay chooses three kinds of disorders: Alzheimer's disease (AD), Phobia, and Autism Spectrum Disorder, which can cause a lot of trouble in people's daily life. In this essay, we are to study the occurrence of some nerve systems' disorders and hope to study the genetic and environmental contribution of these disorders. These three disorders gain a lot of attention as they are very common, and they can partly reflect the contribution of genes and the environment.
\end{abstract}

\section{Instruction}

There are many common disorders without a clear explanation of how the conditions are developed. Studies have been conducted to study the pathology of these disorders. People compare the difference between people with or without the disorder by focusing on environmental and genetic risk factors contributing to these disorders and cause the different behaviors among people with the particular disorder. Environmental risks have a significant impact on human health, either directly or indirectly. People can be exposed to harmful agents, and the ecosystem can be destroyed. Without understanding the pathology of disorders fully, it is difficult to emerge with ideas for treatments. Twin studies and family studies have been invented to identify crucial environmental or genetic effects on a trait. Alzheimer's disease (AD), Phobia, and Autism Spectrum Disorder (ASD) are disorders that gain a lot of attention because of the high diagnostic rate. At the same time, the cause of these disorders is not completely understood.

Alzheimer's disease is a nerve-system disease with aging. The amyloid protein sinks in the brains of the patients so that their nerve cells lose their functions, resulting in conditions where many patients begin to lose the abilities to learn new things and suffer from bad memory. According to prior studies, it's a high heritable disease, making it difficult to treat and cure. However,
Alzheimer's disease is not unchangeable. Some environmental factors can alleviate the level of Alzheimer's disease. And as Alzheimer's disease is also a cognitive disease, it's linked with other cognitive activities like the educational level and complexity of work.

Phobia is a mental disorder that familiar with fear. It is generally thought that the environment creates fear or phobia because of the lack of pathology changes. However, some researchers have proved that genes are important as well. Also, how to cure phobia efficiently is a question that deserved to be discussed.

ASD is a developmental disability that children with ASD often on difficulty communicating with others. It is also a spectrum disorder, and one explanation of why the symptoms of ASD are various is because both genetic and environmental risk factors contribute to the development of ASD. Previous studies have been focusing on the environmental impact of ASD. However, the genetic relationship with the symptoms of ASD needs further study. This review will introduce the environmental factors and genetic factors that result in Alzheimer's disease, Phobia, and ASD.

This review includes some of the known environmental and genetic factors that are related to symptoms of ASD, treatments that are wide-used, and cooccurring disorders. However, this review is not detailed enough to identify the factor that impacts ASD most. More factors contribute to the development of ASD. However, these factors are only risk factors that are not accurate

* Corresponding author: xinzilan@hust.edu.cn 
enough to diagnose ASD. It is important to educate adult how to avoid giving birth to an ASD child, and how to raise an ASD child if they have a child with ASD, which is why more studies are needed on both what lead to ASD, and how if they show more changes in their behaviors and neuronology.

\section{Alzheimer's disease}

\subsection{The basic information of Alzheimer's disease}

\subsubsection{The definition of Alzheimer's disease}

Alzheimer's disease (AD) is a disorder that bothers many old people, and it's very common in old people. This disease is prevalent among people who are 65 years old and older [1]. Alzheimer's disease is linked with nerve system degeneration, A $\beta$ protein accumulation, lipid homeostasis, and immune function [2]. The symptoms of Alzheimer's disease consist of memory problems, cognitive disorders, and difficulty in concentrating on something.

\subsubsection{The symptoms of Alzheimer's disease}

Alzheimer's disease can be divided into three stages. In the beginning, which is the first stage, the patients with $\mathrm{AD}$, usually some old people, always find it hard to remember some tiny things in daily life, even the things that have happened in just seconds. In addition, they have difficulty concentrating on one thing and learn some new things. Sometimes they become bad-tempered and stubborn, and they can form some illusion as their brain can produce some error information. In the middle stage, the patients with $\mathrm{AD}$ can forget some of their practiced skills and have difficulty learning new things. In the final stage, the patients with AD can lose the basic life skills and can only stay in bed.

\subsubsection{The occurrence of Alzheimer's disease}

The Center for Disease Control estimates that the number of people over 65 can rise from 420 in 2000 million to 1 billion in 2030, which means that there may be more old people who can suffer from AD. It's projected by Census data that there will be about 13.8 million people diagnosed with $\mathrm{AD}$ dementia. And at present, there are approximately 36.5 million people in the world suffer from dementia, and most of the cases are AD. And it's estimated that 5-7 million people are diagnosed with AD each year. However, although the number of AD cases is increasing, the rate of $\mathrm{AD}$ in the population is declining. The rate of dementia prevalence has decreased from $11.6 \%$ to $8.8 \%$ after a decade as the increased levels of education among the population [2].

\subsection{The heritability of Alzheimer's disease}

The heritability of Alzheimer's disease is estimated at 0.6, according to the essay called The Role of Heredity in Late-
Onset Alzheimer Disease and Vascular Dementia. In the research, the scientists use the twin study to study a sample of 72 twins whose mean age is 80 years old from 26000 twins pairs in the Norwegian Twin Register. And they compare the resemblance between the dizygotic twins and monozygotic twins to study the genetic impact on Alzheimer's disease. And they find that the pairwise concordance rate for Alzheimer's disease was 78\% (7/9) among monozygotic and 39\% (9/23) among dizygotic twin pairs. And the probands concordance rate was $83 \%$ (10/12) among monozygotic and 46\% (12/26) among dizygotic twin pairs. And there was no significant difference in the apolipoprotein $\mathrm{E} \in 4$ allele rate between twin pairs concordant and discordant for Alzheimer's disease. In the result above, the scientists could find that the concordance rate in monozygotic twins was higher than that of dyzygotic twins. So they concluded that the genetic impact mattered a lot in the occurrence of Alzheimer's disease as monozygotic twins are more similar than dizogotic twins in this trait. And the scientists in the research estimated that the heritability of Alzheimer's disease was about 0.6[3], which means that genes cause $60 \%$ of Alzheimer's disease among the population.

Another research aiming at explaining the role of gene and environment in $\mathrm{AD}$ is done by Margaret Gatz et al. They found that the gene impact contributes largely to the occurrence of the AD as they found that the resemblance in $\mathrm{AD}$ between the monozygotic twins (MZ) was greater than that of dizygotic twins, as the time of onset of $A D$ was more close in MZ than DZ. From their result, three models, including the full model without adjusting for age, the full model adjusting for age, or the reduced model adjusting for age, revealed that the additive genetic influence mattered the most. And in this research, they found that the heritability differs from men to women. It was found that women were more sensitive to the risk of AD than men. From the figure provided, the proportion of genetic impact contributed to the trait of women were more than that of women in three models provided $(0.59$ for women and 0.37 for men in full model without adjusting for age, 0.58 for men and 0.45 for full model adjusting for age. And it was easy to see that when age was considered, the model for men and women can be equal. So scientists can evaluate that the heritability of AD was about 0.5[4], which was similar to research done in The Role of Heredity in Late-Onset Alzheimer Disease and Vascular Dementia.

Also, other research results are reflecting the heritability of AD, which are shown in table 1. In the New York State Psychiatric Institute study, they choose twins from 63 to 73 years old, who are so young, and they found a relatively low heritability based on the resemblance of $\mathrm{MZ}$ is $21 \%$ while the resemblance of DZ is $11 \%$. While in the Norwegian Twin Registry, the concordance rate of $\mathrm{MZ}$ is about $83 \%$, while that of DZ is around $46 \%$. Through these data, it was calculated that the heritability was around $58 \%$. And the scientists of the Finnish Twin Registry found that the concordance rate of $\mathrm{MZ}$ and $\mathrm{DZ}$ are $31 \%$ and $9 \%$ for each. Comparing the concordance rate with that in the Norwegian Twin Registry, it can be 
estimated that the heritability can be similar. And in the subsample of the Swedish Twin Registry based on the SATSA, the heritability is estimated to be 74\%[4].

In conclusion, the heritability of AD is estimated to be around 0.6 considering all the results above, which is higher than 0.5. That is to say, the gene impact matters a lot in $\mathrm{AD}$.

Table1. A Variety of Figure in Many Researches

\begin{tabular}{ccc} 
RESEARCH\RESEMBLANCE & MZ \% & DZ \% \\
\hline $\begin{array}{c}\text { A. L. Mina Bergem et al.'s twin } \\
\text { study }\end{array}$ & 83 & 46 \\
Margaret Gatz et al.'s research & $\begin{array}{c}45 \text { for } \\
\text { men } \\
61 \text { for } \\
\text { women }\end{array}$ & $\begin{array}{c}19 \text { for } \\
\text { men } \\
41 \text { for } \\
\text { women }\end{array}$ \\
$\begin{array}{c}\text { New York State Psychiatric } \\
\text { Institute study }\end{array}$ & 21 & 11 \\
Norwegian Twin Registry, & 83 & 46 \\
Finnish Twin Registry & 31 & 9
\end{tabular}

Table 1. The leftmost column is concerning the research, and the middle column concerns the resemblance in monozygotic twins and the resemblance in dizygotic twins. In Margaret Gatz's study, they classified the resemblance of AD into women and men[3, 4].

\subsection{The environmental impact of Alzheimer's disease}

\subsubsection{Exercise and diet}

Obesity is linked with many diseases like hypertension, strokes, and diabetes, which can play a direct role in the occurrence of $\mathrm{AD}$, especially type 2 diabetes. Insulin resistance can be an index of Alzheimer's disease. AD patients can display a higher brain insulin resistance than normal individuals. And physical activity and diet can have an impact on diabetes and can help protect people from obesity and therefore can prevent people from Alzheimer's disease. Diet can also help reduce the risk of Alzheimer's disease [2].

\subsubsection{Mental demanding activities}

Some demanding mental activities can also function as reducing the risk of suffering from AD. These activities include the level of education and the complexity of work. Some people with low education can be easier to fall into $\mathrm{AD}$. In the classical case-control analysis, the proportion of $\mathrm{AD}$ cases with low education is $88.3 \%$, while the rate of control is about $77.3 \%$. And in the research of Margaret Gatz et al., they found that the odds ratio between education level and risk of Alzheimer's disease is significant in the case-control study. In contrast, the odd ratio between education level and risk of AD in a matchedpair study is not significant, partly because of the high similarity of twins in education. Another intelligent factor is the complexity of work. In the research of Ross Andel et al., they classify the complexity of work into three aspects: work with people, things, and data. And in the case-control study, they found that the complexity of people is protective against AD. And the complexity of things is associated with the low risk of $\mathrm{AD}$ only. When the complexity of work with data and things is considered, people's complexity is only protective against AD [1].

\subsubsection{Stress}

Stress is an important factor that can greatly impact the immune systems, which can help explain the link between stress and $\mathrm{AD}$. Although the relationship between stress and $\mathrm{AD}$ is not clear, it's possible that the stress and the AD can worsen each other, acting as a positive feedback loop. That is to say, the psychological well-being which can be negatively affected by stress can play an important role in the risk of $\mathrm{AD}$. In return, the $\mathrm{AD}$ can influence the brain to produce some error information to affect people's emotions, resulting in the anger and anxiety of the patients, which can contribute to the pressure of people [2].

\subsection{The link between Alzheimer's disease between other traits}

\subsubsection{The link between Alzheimer's disease with the complexity of work}

In another study, scientists think that the high complexity of work may reduce the high risk of Alzheimer's disease. The scientists think that the complexity of work may exercise and reflect people's cognitive ability. They also use two designs, called case-control study and cotwin study, to study the association between the complexity of work and risk of Alzheimer's disease. The sample they use is the Swedish Twins Registry who is 65 years old of age or older in 1998. And to measure the complexity of work, they divide the work into three categories: things, data, and people, and they give an individual complexity to each measure. And the result, which is shown in Table 2, was that in the control study, the low complexity of work with things and the high risk of dementias is significant $(\mathrm{p}=0.05)$ but not for AD. Also, the high complexity of work with people was associated with a reduced risk of all types of dementia and AD only. And when the complexity of work with things and data is considered, the high complexity of work with people can be more significant in protecting people from $\mathrm{AD}$ only. (odds ratio or OR $1 / 40.78 ; 95 \%$ confidence interval or CI $1 / 40.63,0.98)$. And in the cotwin study, Twins with higher scores for the complexity of work with people were at a lower risk of dementia and AD only than their cotwins, and twins with higher scores for the complexity of work with data were at a lower risk of AD. The scientists found that the high complexity of work can effectively reduce the risk of $\mathrm{AD}$, as it may influence the entire intelligence level and the social involvement. The complexity of work with people can help people meet their social requirements, which is more intensive in $\mathrm{AD}$ patients than normal people. Also, another possibility of this phenomenon is that the people with higher complexity of work may have higher social status, which means that 
it can help these people get access to better medical resources to prevent $\mathrm{AD}[1]$.

Table2. Case-Control Analyses and Cotwin Control Analyses of Association Between Occupational Complexity and AD Only

\begin{tabular}{cccc}
\hline Variable & $\begin{array}{c}\text { OR }(95 \% \mathrm{CI}) \text { in } \\
\text { A }\end{array}$ & $\begin{array}{c}\text { OR adjusted for } \\
\text { education } \\
\text { CI })\end{array}$ & $\begin{array}{c}\text { OR }(95 \% \mathrm{CI}) \text { in } \\
\mathrm{B}\end{array}$ \\
\hline $\begin{array}{c}\text { Complexity } \\
\text { with data } \\
\text { AD }\end{array}$ & $0.94(0.85-1.05)$ & $0.98(0.88-1.10)$ & $0.17(0.15-0.57)$ \\
& & & \\
$\begin{array}{c}\text { Complexity } \\
\text { with people } \\
\text { AD }\end{array}$ & $0.80(0.68-0.93)$ & $0.83(0.70-0.98)$ & $0.05(0.01-0.35)$ \\
& & & \\
$\begin{array}{c}\text { Complexity } \\
\text { with things } \\
\text { AD }\end{array}$ & $1.06(0.98-1.15)$ & $1.06(0.98-1.15)$ & $1.11(0.45-2.73)$ \\
& & &
\end{tabular}

Table 2. $\mathrm{AD}=\mathrm{Alzheimer's}$ disease, $\mathrm{OR}=\mathrm{odds}$ ratio, $\mathrm{CI}=$ confidence interval. The data consisted of 146 cases of $\mathrm{AD}$, and $95 \%$ confidence intervals correspond to $\mathrm{p}<0.05$, and ORs were adjusted for age and gender. $A=$ case-control analyses of the association between the complexity of work and $\mathrm{AD}, \mathrm{B}=$ cotwin control analyses of the association between the complexity of work and $\mathrm{AD}$, but the figures were crude [1].

\subsubsection{The link between Alzheimer's disease with the education}

Some scientists found that many patients with low education were more likely to suffer from a high risk of Alzheimer's disease. Therefore, the scientists intended to determine whether there is an association between high education and Alzheimer's disease. They use case-control studies and match-pairs studies to study the association. Their sample is from members of the Study of Dementia in Swedish Twins and members of the OCTO-Twin study. And to measure the level of education, they divided education into two categories: elementary or less schooling and greater than elementary schooling as the sample were old people whose ages were about 80 years old. In Sweden in the 20th century, 6 years of elementary education was mandatory, so virtually no people were not receiving elementary education. And they also take the age, social status, and economic background of the twins into account. Their analysis method was that they estimated the association between education and disease through the odds ratio for the case-control study, comparing proportion with low education in cases and controls. They also use a logistic regression model to adjust for age band and gender. And the results of the casecontrol study are shown in Table 3. However, when dealing with the match-pairs study, they used McNemar's test to estimate the odds ratio with $95 \%$ confidence intervals. And the results of the case-control study are shown in Table 4. And finally, they found that low education is a significant factor in a case-control study that can increase the risk of Alzheimer's disease. However, in the match-pairs study, no odd ratios were significant between high education and dementias. This difference might be explained by the fact that the education level reflects the intelligent reserve, which can influence the risk of $\mathrm{AD}$. And in the match-pairs analysis, the twins may experience a similar environment, resulting in similar intelligent reserve [5].

Table3. Case-Control Analyses of Association Between Education and AD

\begin{tabular}{ccccc}
\hline Cases & $\begin{array}{c}\text { The } \\
\text { proportion } \\
\text { of cases } \\
\text { with low } \\
\text { education }\end{array}$ & $\begin{array}{c}\text { The } \\
\text { proportion } \\
\text { of controls } \\
\text { with low } \\
\text { education }\end{array}$ & $\begin{array}{c}\text { OR } \\
(95 \% \\
\text { CI) }\end{array}$ & $\begin{array}{c}\text { OR }(95 \% \\
\text { CI }) \\
\text { adjusted } \\
\text { for age } \\
\text { and } \\
\text { gender }\end{array}$ \\
\hline $\begin{array}{c}\text { Prevalent } \\
\text { cases(a) } \\
\text { AD }\end{array}$ & $68 / 77$ & $119 / 154$ & 2.22 & 2.26 \\
& & $(77.3 \%)$ & $4.84)$ & $(1.02$, \\
$\begin{array}{c}\text { Incident } \\
\text { cases(a) }\end{array}$ & $50 / 54$ & $92 / 108$ & 2.17 & 2.17 \\
AD & $(92.6 \%)$ & $(85.2 \%)$ & $(0.70$, & $(0.69$, \\
\hline
\end{tabular}

Table 3. In the leftmost column, $a=$ case-control analyses, meaning that the prevalent cases or the incident cases were done in the case-control study, $\mathrm{AD}=$ Alzheimer's disease, $\mathrm{OR}=$ odds ratio, $\mathrm{CI}=$ confidence interval. And $95 \%$ confidence intervals correspond to $\mathrm{p}<0.05$ [5].

Table4. Matched-Pairs Analyses of Association Between Education and $\mathrm{AD}$

\begin{tabular}{|c|c|c|c|c|}
\hline Cases & $\begin{array}{c}\% \text { cases } \\
\text { with } \\
\text { low } \\
\text { education }\end{array}$ & $\begin{array}{c}\% \text { partners } \\
\text { with } \\
\text { low } \\
\text { education }\end{array}$ & $\begin{array}{l}\text { OR } \\
(95 \% \\
\mathrm{CI})\end{array}$ & $\begin{array}{c}\text { Total } \\
\text { number } \\
\text { of pairs }\end{array}$ \\
\hline $\begin{array}{c}\text { Prevalent } \\
\text { cases(b) } \\
\text { AD }\end{array}$ & 87.5 & 83.3 & $\begin{array}{l}2.00 \\
(0.37 \\
10.92)\end{array}$ & 48 \\
\hline $\begin{array}{c}\text { Incident } \\
\text { cases(b) } \\
\text { AD }\end{array}$ & 92.9 & 85.7 & $\begin{array}{l}4.00 \\
(0.45 \\
35.79)\end{array}$ & 42 \\
\hline
\end{tabular}

Table 4 . In the leftmost column, $b=$ matched-pairs analyses, meaning that the prevalent cases or the incident cases were done in the matched-pairs study, $\mathrm{AD}=$ Alzheimer's disease, $\mathrm{OR}=$ odds ratio, $\mathrm{CI}=$ confidence interval. And $95 \%$ confidence intervals correspond to $\mathrm{p}<0.05[5]$.

\section{Phobia}

Phobia is a psychological disorder which often mixed up with fear. It is estimated that more than $40 \%$ of the general population suffers from one or more fears of a specific object or situation at some time in their lives [6]. However, there is a clear border between these two concepts. A fear is termed a phobia when it becomes unreasonable or excessive [7]. People may fear the same thing because of culture, religion, or superstitions. But do biological relatives always fear the same thing? The following part will give some basic information relates to phobias' 
treatment, review several twin and family studies held in different countries and regions, and discuss the genetic and environmental influences in phobia.

\subsection{Treatment}

Many may consider that phobia is a normal phenomenon, which all humankind could have and cued. This opinion is recently proved not that right in research of preparedness theory. This theory proposes that fear is selective and can be extinct. The research group found only 31\% of candidates increased resistance to extinction of conditioned responses to snake and spiders, which means preparedness theory needs more evidence to be admitted, and phobia is probably incurable.[8]But, against this undelightful conclusion, the WHO World Mental Health (WMH)surveys in 24 countries interviewed more than one hundred thousand people. About $23 \%$ of interviewees reported receiving helpful treatment from the first professional seen. While professional treatment increases, the cure rate ends up at a high level of $85.7 \%$. Increasing patient persistence in help-seeking after earlier unhelpful treatments will increase the helpfulness of specific phobia treatment[9].

\subsection{Correlation}

To study systematically, specific phobia is distinguished into five main categories or subtypes by DSM-IV-TR: animal type, natural environment type, situational type, blood-injury-injection type, and other types. The very first twin study about phobia was taken place in Australia in 1998[10]. This research focused on people who suffered from blood phobia and blood faint. The univariate analyses suggested that blood faint and phobia have genetic relevance, and the heritability is 0.71 . It seems that we can conclude this certain type of phobia. But this number was increased by blood faint's influence due to the analysis model. Another research-based on Norwegian clinical [11] in 2000 showed the importance of the environment. Even the lowest environmental of animal phobia was more than 0.5 , dramatically found an opposite result than the former one. It's hard to tell which one is more convincing because the Norwegian study concluded less than 70 pairs of twins, which is too small to be a study sample. So the only method left to solve the problem is doing more studies. The following 3 studies had complex phobia categories and a large sample size. Kendler, Gardner, Annas, and Lichtenstein[12] found an average number of two-fifths of heritage is genetic. Distel's group studied more than 10 thousand pairs of twins in different generations[13]. They limited the heritability lower than 0.1 in blood-injury phobia. And in 2010, Vassend's group did research based on the Norwegian population again[14], and finally, the environment was still more than half

\subsection{Environmental influences}

Besides genetic and environmental influences, shared or non-shared environmental influences can also be distinguished [15]. The non-shared environment seems to greatly influence phobias, but shared environmental influences are not that efficient. For instance, two familiar research drew different, even opposite conclusions. The research about 1106 pairs of twins in 2000 showed significant [16] while another in 2006 showed nothing [17].

The distinction between shared and nonshared environmental influences is relevant to studies of $\mathrm{G} \times \mathrm{E}$. Indeed, most twin analyses do not estimate $\mathrm{G} \times \mathrm{E}$, and if positive interactions between genetic and shared environmental influences are present but ignored, they artificially enlarge estimates of genetic influence. In contrast, positive interactions between genetic and nonshared environmental influence are estimated as a nonshared environmental influence[18].

\subsection{Discussion}

The present work provides a state-of-the-art overview of the available evidence on the heritability of specific phobias and fears. It is a dissatisfying observation that data on the genetic contribution to fears and specific phobias are still scarce [19]. All the researchers who devoted their time to this career look forward to the biological evidence of phobia. There are still much more improvements that can be made in a twin study. Quantifying the standard of fear to differ the fear or searching for a wider array of fear may help enlarge the probability of finding interaction. Enlarge sample size to make higher confidence level. No people are doing a study about phobia in China. I believe that the population in China would be very strong evidence.

\section{Autism Spectrum Disorder}

\subsection{Basic Information}

Autism Spectrum Disorder or ASD is a neurodevelopmental disorder resulting from overall brain reorganization beginning early in development. ASD is one subgroup of the category of pervasive developmental disorders (PDDs), and it's a common condition that affects about $1 \%$ of the population [20]. It is usually more common among men than women. The male to female ratio in the ASD community is close to $3: 1$ [21]. Besides, the disability of a child creates a spillover effect, decreasing the quality of life for the entire family" [22], which is why it is important to focus on the diagnosis and treatment of ASD. ASD causes approximately \$268 billion economic burdens in 2015 because of the educational, medical, and social support provided to the ASD community. Diagnoses of ASD that are wild used today are usually based on observing behaviors. Scientists have constantly put effort into studying biomarkers of ASD. However, no biomarker can be used for early diagnosis and curation of ASD. Some signs of ASD include impairments in social communication, restricted, repetitive, and stereotyped behavior patterns. Children diagnosed with ASD show a mean delay of three years in things, including language development. ASD children tend to play alone, and they can have difficulty 
comprehending other's behaviors and forming friendships months. Table 5 [24] collected potential red flags for ASD from 12 months to 24 months old. The mechanism of ASD remains unclear. Many studies focus on genetic and environmental contribution to the development of ASD, which is why many of the studies are twin studies [25].

Table5. Potential red flags for ASD from 12 months to 24 months old

Potential red flags for autism Potential red flags for autism spectrum disorder for children 12 to 18 months spectrum disorder for old children 18 to 24 months old

Lack of appropriate gaze

Trouble understanding other people's feelings

Lack of warm, joyful expressions with gaze

Difficulty talking about their feelings

Lack of sharing enjoyment or interest

Delayed speech and language skills, including language regression or loss of words

Lack of response to name

Repeats words or phrases (echolalia)

Lack of coordination of gaze, facial expression, gesture, and sound

Lack of showing gestures

Unusual prosody (i.e., inability to vary pitch, loudness, tempo, and rhythm in speech)

Repetitive movements or posturing of body, arms, hands, or fingers

Repetitive movements with objects

Upset by minor changes and does not handle change well

Flaps hands, rocks body

back and forth or spins circles

Interest in watching objects spin

Unusual reactions to sound, smell, taste, or feel [23]. Some signs of ASD can be noticed around 11-12

$\mathrm{ASD}[26]$. The factor contributing most to ASD is genetic $-74 \%$ to $93 \%$ of ASD risk is heritable. Some ASD results from rare genetic syndromes, such as fragile X syndrome 81 and tuberous sclerosis[27]. There have been over 100164 genre and genomics regions associated with ASD (Brugha et al., 2011). The study done by Frazier et al. shows that extreme levels of quantitatively measured autism symptoms were strongly heritable with no significant environmental impact. Less extreme autism symptom levels show lower heritability. Traits such as language ability reported to be greatly affected by heritability. In identical twins, if one twin is diagnosed with ASD, the chance of the co-twin having ASD is 36\%$95 \%$, compared to how the chance is only $0-30 \%$ in nonidentical twins. More than $30 \%$ of ASD cases have resulted from De novo mutations[28]. Mutation of one gene can influence the function of other ASD genes, which means one mutation can be connected to many other mutations through gene regulatory networks. Individuals with de novo mutations tend to have lower non-verbal $\mathrm{IQ}[28]$. Some of the deletion and duplication occur because of rare variants inherited from a mother or father. Recessive variants become helpful to identify ASD cases in consanguineous families[28]. Common polygenic risk scores (PRS) include "the contribution of all common Single nucleotide polymorphisms (SNPs) to a trait"[28]. Brainstorm Consortium et al. found a relationship between PRS and higher educational attainment and IQ in the ASD population.

Avoids or resists physical contact

Flat or inappropriate facial expressions

Talks in a flat, monotone, or sing-song voice

Lines up toys or other objects

Failure of back-and-forth conversation

Failure to initiate or respond to social interactions

\subsection{Genetic Influences}

Although the pathogenesis of ASD does not know, early twin studies have supported a strong genetic etiology to

\subsection{Environmental Influences}

ASD is a result of both genetic influences and environmental influences, which is why ASD is a spectrum disorder with a range of linked conditions, and why $40-50 \%$ of ASD cases are different. Some environmental risk factors of ASD include short interpregnancy intervals ( $<24$ months) and non-optimal factors during pregnancy such as maternal metabolic conditions, weight gain, and hypertension. Preterm birth ( $<32$ weeks), low birth, weight $(<1500 \mathrm{~g})$, small-for-gestational-age status, and large-for-gestational, was used to calculate the SRS relationship [29]. Advanced maternal age can relate to de novo mutations and epigenetic alterations, which is why advanced maternal age is positively associated with the risk of autism in the offspring. Wu et al. found that each increase of 10 years in maternal age will relate to 18 percent high risk in developing ASD, and an increase of 10 years in paternal age relates to an increase of risk of developing ASD by 21 percent. Lower maternal age is associated with lessening the risk of autism in children by over 10 percent, and lower paternal age was associated with reduced risk by nearly $20 \%$ percent. "Higher maternal age was associated with $41 \%$ increased risk of autism, and higher paternal age was associated with 55\% increased risk." [30].

\subsection{Treatment}

The treatments of ASD that are widely used are behavioral interventions aiming to make the behavior symptoms less 
severe. Some examples include Early Social Interaction (ESI), Parent-Child Interaction Training (PCIT), and Reciprocal Imitation Training (RIT). ESI is a multisite toddler treatment that can be given to both the parents and the ASD child. ESI helps parents support their child's social communication, emotional regulation, and play in everyday activities and settings. It offers interventions that support the child with the development of social communication skills. PCIT was a treatment for all types of children that was aimed to treat maladaptive behaviors that interfere with learning. RIT was developed to help ASD child to learn how the imitate people. Since people's behaviors change more easily in childhood, early therapy means the children can receive help early in life which improves the effectiveness of the therapy. The benefit of early treatment brings up the need for early diagnosis. Screening of Autism has been hard due to "insufficient time to screen, unfamiliarity with screening tools, lack of reimbursement, and cost of screening tools" [24].

\subsection{Co-occurring disorder of ASD}

ASD without coexisting disorders is extremely rare because ASD shares "substantial etiological factors with other disorders" [25]. Co-occurring disorders for ASD include two types: classic medical problems and mental health conditions. Examples of classic medical problems include epilepsy, gastrointestinal issues, and sleep disorders. Mental-health conditions related to ASD include attention deficit hyperactivity disorder (ADHD), depression, obsessive-compulsive disorder, or depression. Soke et al. identified 8 disorders that have a statistically significant relationship with ASD. They are DHD, oppositional defiant disorder, anxiety disorder, aggression, language disorder, sleep abnormalities, motor disability, and mood problems. Allergies, gastrointestinal disturbance (GID), and seizures are usually common among families who have individuals diagnosed with ASD [31]. With the help of a twin study, scientists can compare co-twins of a person who has been diagnosed with ASD and calculate the percent of the co-twin having coexisting disorder with or without ASD [25].

\section{Conclusion}

These three diseases are bothering a lot of people, and it's common in our daily life. Alzheimer's disease tends to happen with aging, and Phobia happens due to certain cultures, religions, or superstitions. Autism Spectrum Disorder can be detected at a young age and is a developmental disorder. The previous study discovered the biological relationship between these three kinds of mental disorders through methods like twins study. Among them, heritability is not always significant as in the case of phobia. While for ASD and Alzheimer's disease, the risk of both diseases is heritable. Environmental impact cannot be ignored in these three diseases, which means that the environmental factors should be considered even for highly heritable diseases like Alzheimer's disease and Autism Spectrum Disorder. And most of these mental diseases can act with other traits, especially for diseases with high heritability, as different diseases or behavior can share the same environmental factors and genetic factors in pathology. However, behavior genetics analyses can only function as prediction and how to diagnose or predict mental disorders still needs a lot of work to do in the future. But data analysis in genetic and environmental analysis so far still can offer people some advice to avoid some risk of these diseases.

\section{References}

1. Andel, et al., Complexity of Work and Risk of Alzheimer's Disease: A Population-Based Study of Swedish Twins. Journals of Gerontology Series B: Psychological Sciences \& Social Sciences, 2005.

2. Robinson, et al., Recent Progress in Alzheimer's Disease Research, Part 2: Genetics and Epidemiology. Journal of Alzheimer's disease: JAD, 2017. 57(2): p. 317-330.

3. Bergem, A., The Role of Heredity in Late-Onset Alzheimer Disease and Vascular Dementia: A Twin Study. Arch Gen Psychiatry, 1997. 54(3): p. 264.

4. Gatz, M., et al., Role of genes and environments for explaining Alzheimer disease. Arch Gen Psychiatry, 2006. 63(2): p. 168-174.

5. Margaret, G., et al., Education and the risk of Alzheimer's disease: findings from the study of dementia in Swedish twins. Journals of Gerontology, (5): p. P292.

6. Oosterink, F., A.D. Jongh and J. Hoogstraten, Prevalence of dental fear and phobia relative to other fear and phobia subtypes. European Journal of Oral ences, 2010. 117(2): p. 135-143.

7. Association, A.P., Diagnostic and Statistical Manual of Mental Disorders (DSM-IV-TR). 2000: Diagnostic and Statistical Manual of Mental Disorders (DSM-IVTR).

8. Fredrik, et al., Biological preparedness and resistance to extinction of skin conductance responses conditioned to fear relevant animal pictures: A systematic review. Neuroscience and biobehavioral reviews, 2018.

9. A, Y.A.D.V., et al., Perceived helpfulness of treatment for specific phobia: findings from the World Mental Health Surveys. Journal of Affective Disorders, 2021.

10. Testing a genetic structure of blood-injury-injection fears (p377-384). American Journal of Medical Genetics Part B Neuropsychiatric Genetics, 2010. 81(5): p. 377-384.

11. Skre, I., et al., The heritability of common phobic fear: a twin study of a clinical sample. Journal of Anxiety Disorders, 2000. 14(6): p. 549-562.

12. Kendler, K.S., et al., The Development of Fears from Early Adolesence to Young Adulthood: A Multivariate Study. Psychological Medicine, 2008. 38(12): p. 1759-1769. 
13. Distel, M.A., et al., Heritability of Self-reported Phobic Fear. Behavior Genetics, 2008. 38(1): p. 2433.

14. Vassend, O., E. R Ysamb and C.S. Nielsen, Dental anxiety in relation to neuroticism and pain sensitivity. A twin study. Journal of Anxiety Disorders, 2011. 25(2): p. 302-308.

15. Gregory, A.M., J. Lau and T.C. Eley, Finding geneenvironment interactions for phobias. European Archives of Psychiatry\&Clinical Neuroscience, 2008. 258(2): p. 76-81.

16. Lichtenstein, P. and P. Annas, Heritability and Prevalence of Specific Fears and Phobias in Childhood. Journal of Child Psychology \& Psychiatry, 2010. 41.

17. Bolton, D., et al., Prevalence and genetic and environmental influences on anxiety disorders in 6year-old twins. Psychological Medicine, 2006. 36(3): p. 335-44.

18. V., R.F. and P.C. Sham, Analytic approaches to twin data using structural equation models. Briefings in Bioinformatics, (2): p. 119.

19. Houtem, C.V., et al., A review and meta-analysis of the heritability of specific phobia subtypes and corresponding fears. Journal of Anxiety Disorders, 2013. 27(4).

20. Baird, G., et al., Prevalence of disorders of the autism spectrum in a population cohort of children in South Thames: the Special Needs and Autism Project (SNAP). Lancet, 2006. 368(9531): p. 210-215.

21. Loomes, et al., What Is the Male-to-Female Ratio in Autism Spectrum Disorder? A Systematic Review and Meta-Analysis. Journal of the American Academy of Child and Adolescent Psychiatry, 2017.

22. Frye, R.E., et al., Emerging biomarkers in autism spectrum disorder: a systematic review. Annals of Translational Medicine, 2019. 7(23): p. 792-792.

23. Young, R.L., N. Brewer and C. Pattison, Parental identification of early behavioural abnormalities in children with autistic disorder. Autism the International Journal of Research \& Practice, 2003. 7(2): p. 125.

24. Brasher, S. and J.L. Stapel-Wax, Autism Spectrum Disorder in the Primary Care Setting: Importance of Early Diagnosis and Intervention. Advances in Family Practice Nursing, 2020. 2.

25. Lundstrm, S., et al., Autism spectrum disorders and coexisting disorders in a nationwide Swedish twin study. Journal of Child Psychology and Psychiatry, 2015. 56.

26. Bailey, A.J., et al., Autism as a strongly genetic disorder: evidence from a British twin study. Psychological Medicine, 1995. 25.

27. Lord, C., et al., Autism spectrum disorder. Nature Reviews Disease Primers, 2020. 6(1): p. 5.

28. Iakoucheva, L.M., A.R. Muotri and J. Sebat, Getting to the Cores of Autism. Cell, 2019. 178(6): p. 12871298.
29. Modabbernia, A., E. Velthorst and A. Reichenberg, Environmental risk factors for autism: an evidencebased review of systematic reviews and metaanalyses. Molecular Autism, 2017. 8(1): p. 13.

30. Wu, S. Advanced parental age and autism risk in children: a systematic review and meta " nalysis. Acta Psychiatrica Scandinavica, 2016. 135(1): p.29.

31. Aldinger, K.A., et al., Patterns of Risk for Multiple Co-Occurring Medical Conditions Replicate Across Distinct Cohorts of Children with Autism Spectrum Disorder. Autism Research, 2015. 8(6): p. 771-781. 